\title{
Complications in Head and Neck Surgery
}

\section{Prathamesh S Pai}

Associate Professor and Surgeon, Department of Head and Neck Surgical Oncology, Tata Memorial Hospital, Mumbai Maharashtra, India

Correspondence: Prathamesh S Pai, Associate Professor and Surgeon, Department of Head and Neck Surgical Oncology Office 46, Tata Memorial Hospital, Dr Ernest Borges Marg, Parel, Mumbai-400012, Maharashtra, India, Phone: +91 2224177185 Fax: +91 22 24146937, e-mail: drpspai@gmail.com

\begin{abstract}
Surgical outcomes are more or less predictable with a reasonable degree of accuracy. Head and neck surgery has potential for many complications due to presence of a large number of major vessels and nerves. In head and neck surgery, complications can arise due to airway compromise, vascular injury, infections and rarely physiologic causes. Carefully performed surgery is the cornerstone of success. Step by step approach with attention to details and a thorough check after completion of the procedure will ensure optimal results without complications.
\end{abstract}

Keywords: Head and neck neoplasms, complications.

Surgical outcomes are more or less predictable with a reasonable degree of accuracy. None of the procedures being performed in routine head neck surgery are novel and have been perfected over the past century. It is no surprise that most complications have their origin during surgery. Inadvertent failure to perform certain steps or perform checks may result in complications. Having said this there may still be unexplained complications in rare situations.

In Head and Neck surgery, complications can arise due to airway compromise, vascular injury, infections and rarely physiologic causes. In this chapter we will focus on discussion of complications as they happen during commonly perfomed procedures. This chapter is not supposed to be an exhaustive tome on complications but will aim to provide the optimum methods of management of Head and Neck surgical complications.

\section{DURING NECK DISSECTION}

The neck dissection is the most commonly performed surgical procedures and is an important adjunctive procedure to resection of the primary head and neck tumor. When performed well the neck dissection can be an exquisite surgery encompassing muscles, veins, arteries and nerves of varying dimensions and of utmost importance to function and form.

\section{INCISION PLANNING AND SKIN FLAP NECROSIS}

Conforming to the vascular supply and planning of incision in the watershed lines will give us an optimum access to all structures without compromising on skin approximation and healing.

The neck skin is supplied by the facial artery and its branches through the platysma from superior to inferior

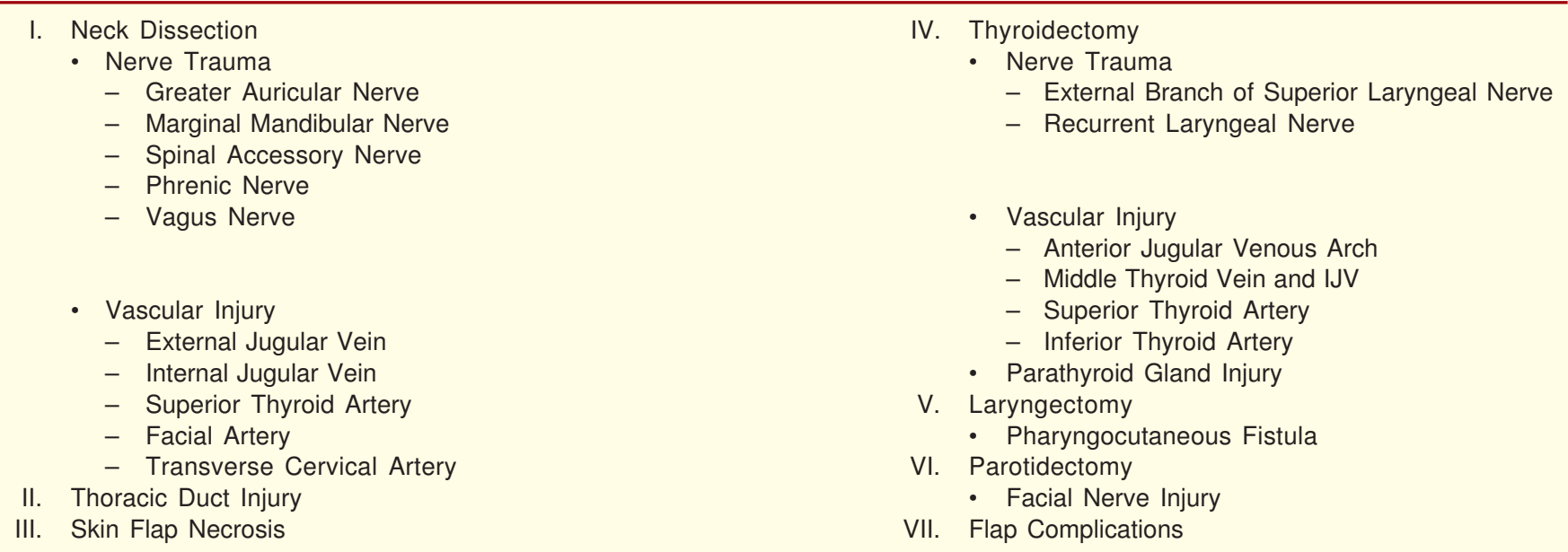


direction while the lower neck is supplied by the transverse cervical artery and its branches. The mid portion and posterior neck skin is supplied by superior thyroid and occipital arteries. Incisions planned transversely and anteriorly placed well within the platysma give the best cosmetic outcome. Single incision without trifurcate point is better. Mcfee incision and the Hockey stick ' $\mathrm{J}$ ' incisions are preferred to avoid tri-pointer flap breakdown. During closure of the neck wound, proper approximation of the platysma ensures complete closure of the neck and proper healing with minimum scarring.

\section{Nerve Trauma}

\section{Greater Auricular Nerve (GAN)}

It has two divisions which innervate the pinna and the skin posterior to it and trauma to this sensory nerve numbs those areas. The GAN lies lateral to the external jugular vein. Both these structures are subcutaneous, do not have protection of the platysma and can be easily damaged in the process of incising the skin. The posterior branch can be sacrificed during parotidectomy after safely identifying and preserving the anterior branch.

\section{Marginal Mandibular Nerve (MMN)}

The MMN which is the upper division of the cervicofacial trunk of the facial nerve exits the parotid fascia and traverses the superficial investing fascia of the neck to innervate depressor anguli oris, the depressor labii inferioris, the inferior fibers of the orbicularis oris and the mentalis muscles. Damage to this nerve can cause salivary incontinence and esthetic impairment due to an alteration in the balance of the musculature around the lower lip, preventing lateral and downwards movements and lower lip inversion. MMN can be identified at the point where it exits from the parotid by dissecting the fascia $1 \mathrm{~cm}$ below and lateral to the angle of mandible. The nerve runs in the fascia over the facial vessels. After dividing the facial vessels they can be retracted upwards moving the nerve out of the path of further neck dissection.

\section{Spinal Accessory Nerve (SAN)}

Spinal accessory is a motor nerve supplying the sternocleidomastoid and the trapezius muscles. During neck dissection it can be identified either at its exit from the jugular foramen where it runs lateral to the internal jugular nerve or at the Erb's point where it can be located $1 \mathrm{~cm}$ posterior and superiorly. Approximately $30 \%$ of patients will suffer from a shoulder dysfunction following handling of the SAN during level II and V dissection. Early postoperative shoulder physiotherapy including both active and passive movements along with progressive resistance exercise training (PRET) is found to be more effective than routine physiotherapy. Patients with advanced shoulder dysfunction cannot be offered much relief due to physical changes such as muscular atrophy, capsular adhesions and fibrosis. Hence it is imperative to initiate shoulder exercises in all patients following a neck dissection.

\section{Vascular Complications}

\section{Intraoperative and Reactive Hemorrhage}

It can be anticipated during neck dissection from vessels in close proximity to lymph node groups. The most common veins injured are the tributaries of the internal jugular vein which are usually arise from structures medial to the IJV, those coming from the thyroid gland (middle thyroid vein), the pharyngeal veins alongside the hypoglossal nerve and the facial vein. The various arteries encountered during neck dissection are the occipital artery at Level II, the superior thyroid artery at Level III and the transverse cervical vessels at Level IV and V lymph nodes. Special attention needs to be focused on these named and other small unnamed vessels during surgery to avoid immediate hemorrhage on reversal of anesthesia when the intrathoracic pressure varies.

An injury to the common carotid needs to be tackled while maintaining blood pressure and patient position to ensure adequate cerebral perfusion. A vascular clamp needs to be used to stem the bleeding and the repair of the vessel carried out with 6-0 nonabsorbable sutures such as prolene.

\section{Secondary Hemorrhage}

Surgery in the head and neck region involves not only neck dissection where one encounters many vessels but also resection of structures in vicinity of the aerodigestive tract. This can lead to communication of the oral cavity with the neck and even the skin forming an orocutaneous fistula. Over time the saliva can contaminate the neck and lead to infection and dissolution of vessel walls leading to hemorrhage manifesting as 'carotid blowout'.

A set protocol needs to be followed to treat a patient with postoperative hemorrhage. The immediate hemorrhage needs to be explored to secure the bleeding vessel following 
which a survey of all named vessels is mandatory. The venous drainage channels should be assessed under positive pressure ventilation to complete the exploration. Special care should be taken of the flaps used for reconstruction to avoid damaging their pedicles.

\section{THORACIC DUCT INJURY}

Chyle drains into the venous system via the thoracic duct. The thoracic duct crosses the root neck posterior to the common carotid artery and drains into the confluence of the internal jugular vein and the subclavian vein. The thoracic duct is present on the left side in normal individuals. Variations have been reported wherein there is a prominent lymphatic channel on the right side as well.

During left neck dissection the thoracic duct is at danger of injury and it is mandatory to check for it by lowering the head end and applying positive pressure ventilation to increase the intrathoracic pressure. This helps identify any latent leaks. If present, it should be under-run with 3-0/4-0 nonabsorbable suture material preferably colored to identify the site of repair in event of a re-exploration for profuse chyle leak.

Most patients with head and neck malignancies are dehydrated and are kept overnight fasting prior to surgery. Both these together might prevent detection of a small thoracic duct injury during surgery. Usually it manifests 48 hours postoperative once the nasogastric feed is initiated. It is imperative to treat it with respect as unchecked the patient may develop hypoproteinaemia, hyponatremia, hypochloremia, lymphocytopenia and an overall immunocompromised status which may impede a successful convalescence and proper wound healing.

Monitor the postoperative neck drain. Suspect chyle leak if there is clear fluid of large quantity beyond 48 hours by which period the drains usually reduce in quantity.

If the quantity is more than $500 \mathrm{ml}$ in 24 hours it would merit an exploration to contain the leak. To increase chances of identifying site of leak, the patient may be given nasogastric tube feed with high fat content 1-2 hours prior to exploration. Multiple sutures placed through surrounding tissue for support help in containing the leak. Local muscle flaps (sternocleidomastoid, omohyoid and levator scapulae) may be rotated into the supraclavicular fossa to seal the region lateral to the carotid artery if there is a generalized leak. If the leak persists, thoracoscopic ligation of the thoracic duct may help.

If the leak is less than $500 \mathrm{ml}$ general measures such as bed rest, head elevation, pressure dressings and gravity aided drainage may contain the leak. Enteral feeding with diet low in long chained triglycerides (LCTs) and high in medium chained triglycerides (MCTs), for example, coconut oil will reduce the chyle quantity as it is directly absorbed via the intestines into the portal circulation.

\section{DURING THYROIDECTOMY}

\section{Laryngeal Nerve Injury}

During thyroidectomy the external branch of the superior laryngeal nerve (EBSLN) and the recurrent laryngeal nerve (RLN) are at risk. Nerve injuries may be temporary or permanent. The incidence of transient temporary RLN injury is around 5\% and permanent injury occurs in 2 to $3 \%$ of cases in most series. The most important cause for permanent RLN injury is division of the nerve. In malignant thyroid disease, every attempt is made to preserve a functioning nerve. However, occasionally the nerve may be sacrificed, if the disease cannot be dissected off the nerve and if it is the only site of residual disease.

Knowledge of anatomy and meticulous surgical techniques is a must. Proper identification of nerves during surgery is documented to reduce the incidence of damage to these structures.

\section{Recurrent Laryngeal Nerve (RLN)}

a. Relation in the Beahr's triangle: RLN forms the third boundary of a triangle formed by the common carotid artery and the inferior thyroid artery low down in the tracheoesophageal groove.

b. Relation to inferior thyroid artery (ITA): RLN lies posterior to ITA in the majority of cases, however, it may lie anterior or traverse between the branches of ITA.

c. Relation to the Zukerkandl's tubercle: Zukerkandl's tubercle is a lateral projection of the thyroid lobe formed by the fusion of the medial and lateral thyroid analges. The RLN lies posterior to this tubercle with the superior parathyroid lying further posterior to the RLN.

d. Relation to the Berry's ligament: The RLN may penetrate the Berry's ligament which is a posterior condensation of the pretracheal fascia binding the thyroid to the cricoids and upper tracheal rings.

e. Relation to the cricothyroid joint: The RLN enters the larynx anteromedial to the cricothyroid joint.

f. Nonrecurrent laryngeal nerve: Seen in $1 \%$ of cases, a RLN may be nonrecurrent and is usually associated with an anomalous subclavian artery. 
Unilateral RLN damage results in a vocal cord paralysis. The symptoms depend on the degree of abduction of the vocal cord. If the vocal cord is paralyzed in the paramedian position voice will be almost normal. If the vocal cord is paralyzed in the cadaveric position the gap between the cords being more results in a whisper with a poor cough reflex. The final position that the cords take is apparent only after 6 months.

Bilateral cord damage can result in both cords being either in the paramedian position or in abduction. If cords are in the paramedian position, it will result in a reduced glottic air space which would necessitate a tracheostomy. On the other hand, if both cords are paralyzed in abduction, the patient will have severe aspiration, which may also necessitate a tracheostomy.

\section{External Branch of the Superior Laryngeal Nerve (EBSLN)}

a. Relation to the Joll's triangle: The EBSLN lies deep to the superior pole of the thyroid gland as it passes to the cricothyroid muscle in the sternothyrolaryngeal (Joll's) triangle formed laterally by superior thyroid pole, superiorly by the attachments of the strap muscles and medially by the midline.

b. Relation to the superior thyroid pole: Depending on the distance of the EBSLN from the superior pole of the thyroid gland, Cernea et al, proposed a classification given below. In their study, there was more than $50 \%$ incidence of the EBSLN being less than $1 \mathrm{~cm}$ from the superior thyroid pole suggesting need for meticulous dissection of the individual superior thyroid artery branches as close to the gland whilst trying to identify the nerve.

When the EBSLN is damaged unilaterally, it usually goes unnoticed by a normal individual. A trained singer will notice a change in pitch variation, with inability to go to a higher pitch and may affect the singing career. Hence in a professional voice user extreme care should be taken to preserve the EBSLN and the patient should be advised of this possibility. On examination the damaged side will show a bowed vocal cord wrinkly or wavy in appearance and at a lower level than the opposite cord.

Bilateral EBSLN damage causes a lower pitch voice which is weaker and breathy. The singing will be severely affected at higher notes but not the conversational speech.

Caution has to be exercised with use of electrocautery with preferential use of bipolar over unipolar whilst delianeating the nerves and achieving hemostasis. During surgery magnification with loupe or microscope are proved to be better than conventional dissection.

\section{Vascular Injury}

Thyroidectomy is an anatomical surgery when performed optimally can be quite avascular. Many surgeons practice day care surgery wherein a drain is not inserted. This can be done only with meticulous hemostasis. The most common cause for re-exploration following thyroidectomy is hemorrhage. Notorious amongst the vessels are the anterior jugular venous arch and the middle thyroid vein. The veins are identified and preserved as far as possible unless there is a communication present in midline between the anterior jugular veins. The middle thyroid vein has to be sought out early on in the dissection and secured to prevent inadvertent tear of the internal jugular vein. Positive pressure ventilation following completion of surgery can pre-empt venous ooze during reversal of anesthesia.

- Superior thyroid artery (STA) is the main blood supply to the thyroid gland via a lease of vessels around the superior pole. Careful identification of the individual vessels and ligation is recommended. The STA can be identified by delineating the carotid artery and tracing the first branch of it downward towards the superior pole of the thyroid gland. The STA supplies the laryngeal skeleton as well and its branches close to the cricothyroid membrane can bleed whilst dissecting the thyroid gland from the ligament of berry jeopardising the RLN at its entry into the larynx behind the inferior cricothyroid joint.

- Inferior thyroid artery (ITA) a branch of the thyrocervical trunk supplies the thyroid gland alongside the trachea and the esophagus. More importantly the blood supply of the parathyroids is via the ITA. Careful dissection of the thyroid gland in the subcapsular plane will delineate the terminal branches of the ITA which can be ligated. This ensures proper hemostasis and preservation of blood supply to the parathyroid glands.

\section{Parathyroid Injury}

Hypocalcemia calculated as $<2.1 \mathrm{mmol} / \mathrm{L}$ or $9 \mathrm{mg} / \mathrm{dL}$ or an ionized calcium level $<1.1 \mathrm{mmol} / \mathrm{L}$ or $4.5 \mathrm{mg} / \mathrm{dL}$ can be caused by parathyroid injury following thyroidectomy. Postoperative hypocalcemia can be transient (less than 6 
weeks) or permanent (more than 6 months). It can result from vascular damage, direct trauma, inadvertent or intended removal of parathyroid glands during surgery.

Total thyroidectomy has higher rates as compared to subtotal or hemithyroidectomy and the rates are higher when a central neck dissection of the Level VI (tracheaoesophageal groove) is performed. Hypocalcemia presents with signs of generalized neuromuscular irritability including paresthesia, muscle cramps, laryngospasm, tetany and seizures. This neuromuscular irritability can also be displayed through elicitation of Chvostek's (facial muscle twitch) and Trousseau's sign (carpopedal spasm).

Thorough knowledge of the thyroid-parathyroid anatomy and a meticulous surgical procedure ensuring capsular ligation of branches of the inferior thyroid artery as opposed to truncal ligation, may decrease rates of hypocalcemia. In eventuality of inadvertent or planned removal of the parathyroid gland, it can be reimplanted after confirming that the cut tissue is parathyroid gland in frozen section, $1 \mathrm{~mm}$ slices of removed parathyroid gland are prepared and these are inserted into a pocket made in the sternocleidomastoid muscle. In case of extensive neck dissections, one can use pectoralis major for implantation. In cases of surgery for medullary thyroid cancer associated with MEN II A and IIB, forearm is the preferred choice.

Mainstay in management of hypocalcemia is supplementation with calcium and vitamin-D3. It is also essential to measure serum magnesium in any patient who is hypocalcemic, as correction of hypomagnesemia must be done to overcome PTH resistance before serum calcium will return to normal.

Asymptomatic patients can be managed with oral supplements alone. In symptomatic patients to raise serum calcium levels by $2-3 \mathrm{mg} / \mathrm{dL}$, administration of $15 \mathrm{mg} / \mathrm{kg}$ elemental calcium over 4-6 hours is required. Oral calcium supplementation is started concurrently with elemental calcium $15 \mathrm{mg} / \mathrm{kg} /$ day (calcium carbonate 1-3 g/day) and vitamin D3 either Calcitriol (0.25-0.5 mg up to four times daily) or Ergocalciferol (50,000-100000 IU/Day) which is less expensive and has longer duration of action. Evaluation of serum calcium is important as excessive calcium consumption may lead to hypercalciuria with nephrocalcinosis and/or nephrocalcinosis, hence it is mandatory to monitor calcium annually with 24 hours urine calcium $(<4 \mathrm{mg} / \mathrm{kg} / 24 \mathrm{hr})$.

\section{DURING PAROTIDECTOMY}

\section{Facial Nerve Injury}

Facial paresis occurs in $25 \%$ cases following superficial parotidectomy most of which recover with minimal residual weakness. Permanent paralysis occurs in less than $10 \%$ of cases with reduced incidences in high volume centers.

Thorough knowledge of the extra temporal course of the facial nerve and meticulous surgical technique while identifying it at the stylomastoid foramen will prevent nerve injury. Most injuries are the result of extensive dissection resulting in nerve handling and devascularization.

If facial nerve structural integrity is certain and there is paresis, then conservative treatment is initiated whilst waiting for facial nerve regeneration after Wallerian degeneration which takes approximately $1 \mathrm{~mm}$ per day. During this period corneal protection is mandatory with either temporary tarsorraphy or gold implants.

If facial nerve is transacted immediate repair gives best results. The various methods to repair the nerve are:

1. End to end anastomosis if proximal and distal stump are seen and a tension free anastomosis is possible can be achieved.

2. Interposition or cable graft if a section of the nerve has been excised and primary suturing not possible because of nerve loss. The various nerves used are greater auricular nerve, sural nerve, ansa hypoglossi and medial antebrachial cutaneous nerve.

3. If proximal stump is unavailable, Hypoglossal - facial nerve anastomosis can be done.

\section{FOLLOWING LARYNGECTOMY}

\section{Pharyngocutaneous Fistula}

PC fistula is the most common complication following laryngectomy. Fistulae generally occur in the immediate postoperative period anytime between 5-15 days. However, occurrence of late fistulae especially during or after adjuvant therapy is also known. Factors which predispose to PC fistula are poor nutritional status, low serum albumin, anemia, comorbidities such as diabetes, prior chemoradiotherapy and large pharyngeal defects.

Surgical technique is of utmost importance in ensuring prevention of a PC fistula. The type of suture material (absorbable versus nonabsorbable), type closure (horizontal versus vertical), pattern of closure (continuous vs interrupted) are not known to alter leak incidence. 
Meticulous tension free closure is the most important factor in ensuring a smooth convalescence. In patients who have been salvaged following chemoradiation all care should be taken to ensure a proper pharyngeal closure, augmenting with a vascularized flap if the defect is large and avoiding tension at all costs. If the pharynx is closed primarily a pectoralis major myofascial flap is still used to cover the neopharynx which will provide a vascularized cover for the vessels and also help contain a leak and protect the major vessels.

In the immediate postoperative period, a pharyngeal leak will manifest with edematous skin flaps and erythema. The skin flaps will appear loose and the drain will show flecks of organic material and air bubbles. Patient may also manifest fever, hemorrhage and wound breakdown.

If there are signs of leak within 48 hours or there is massive drain output a technical failure of pharynx reconstruction is the cause and it merits immediate exploration and closure of the pharynx along with augmentation using a pectoralis major myofascial flap. Any bleeding from the neck associated with a leak requires an exploration.

If there are signs of minimal leak then a conservative line of management is followed. Nasogastric feeding is continued along with antibiotics. The leak is lateralised by placing corrugated drains (more efficient than vacuum drains) to prevent passage of saliva into the tracheostome. In the event of salivary aspiration into the trachoestoma a cuffed tracheostomy tube with supra cuff suction aid should be inserted.

\section{FLAP COMPLICATIONS}

\section{Pedicled Flaps}

The workhorse of reconstruction remains Ariyans' pectoralis major myocutaneous flap. It is used extensively in reconstructing the oromandibular defects, patching the pharynx, providing cover for the carotid artery and at times resurfacing the skin. It is a pedicled flap and dependant on the blood supply below the clavicle. The skin overlying the muscle is supplied by the perforators. The complications occur especially in obese individuals and females with large amount of subcutaneous fat on the chest wall. The skin island can get sheared off or the site of fixation can give way due to weight of the flap and maceration of the recipient site mucosa leading to an orocutaneous fistula. This can be avoided by meticulous suturing of the island of skin to the underlying muscle. The flap needs to be held in place by anchoring to a bone either the mandible or the maxilla.

\section{Free Flaps}

Free tissue transfers have revolutionized reconstruction. With free tissue we ensure good vascularity and composite tissues which match the requirement. Free flaps also heal faster allowing proper completion of planned treatment. These flaps require higher technical skills and are dependent on recipient vessels. Flap monitoring vital to assess vascularity in the immediate postoperative period is done clinically by response to prick by hypodermic needle. It is most common and reliable. Normal color, temperature, absence of congestion or edema and slow bright red blood on needle prick is the sign of adequate perfusion of flap. A flap with venous congestion is edematous, bluish, cold and on needle prick shows brisk and profuse flow of dark blood. A flap with arterial blockade shows loss of tissue turgor, cold, blanched and on needle prick there is no flow of blood. Urgent re-exploration and remedy of the cause of vascular compromise can salvage the flap. There is good correlation between time from detection of thrombosis to re-exploration and chances of survival of flap, i.e. the longer the time between thrombosis and re-exploration, the fewer the chances of successful salvage of flap.

\section{SUMMARY}

Head and neck surgery has potential for many complications due to presence of a large number of major vessels and nerves. Carefully performed surgery is the cornerstone of success. Step by step approach with attention to details and a thorough check after completion of the procedure will ensure optimal results without complications.

\section{SUGGESTED READING}

Books:

1. Thawley SE, Panje WR, Batsakis JG, Lindberg RD. Comprehensive Management of Head and Neck Tumours (2nd ed) Philadelphia: W B Saunders 1999.

2. Conley JJ. Complications of Head and Neck Surgery. Philadelphia: W B Saunders 1979.

Articles-Recurrent Laryngeal Nerve Injury:

1. Hermann M, Alk G, Roka R, et al. Laryngeal recurrent nerve injury in surgery for benign thyroid diseases: Effect of nerve dissection and impact of individual surgeon in more than 27,000 nerves at risk. Ann Surg 2002;235(2):261-68. 
2. Shindo M, Chheda NN. Incidence of vocal cord paralysis with and without recurrent laryngeal nerve monitoring during thyroidectomy. Arch Otolaryngol Head Neck Surg 2007;133(5):481-85.

3. Dralle H, Sekulla C, Lorenz K, et al. German IONM Study Group Intraoperative monitoring of the recurrent laryngeal nerve in thyroid surgery. World J Surg 2008;32(7):1358-66.

4. Cernea CR, Ferraz AR, Nishio S, et al. Surgical anatomy of the external branch of the superior laryngeal nerve. Head Neck 1992;14(5):380-83.

5. Hartl DM, Travagli JP, Leboulleux S, et al. Clinical review: Current concepts in the management of unilateral recurrent laryngeal nerve paralysis after thyroid surgery. J Clin Endocrinol Metab 2005;90(5):3084-88.

6. Franco RA, Andrus JG. Aerodynamic and Acoustic Characteristics of Voice Before and After Adduction Arytenopexy and Medialization Laryngoplasty with GORE-TEX in Patients with Unilateral Vocal Fold Immobility. J Voice 2008.

\section{Facial Nerve Injury:}

1. Finsterer J. Management of peripheral facial nerve palsy. Eur Arch Otorhinolaryngol 2008;265(11):1433-34.

2. Shindo M. Management of facial nerve paralysis. Otolaryngol Clin North Am 1999;32(5):945-64.

\section{Shoulder Dysfunction:}

1. Stuiver MM, van Wilgen CP, de Boer EM, et al. Impact of shoulder complaints after neck dissection on shoulder disability and quality of life. Otolaryngol Head Neck Surg 2008;139(1):3239.

2. van Wilgen CP, Dijkstra PU, van der Laan BF, et al. Shoulder complaints after nerve sparing neck dissections. Int J Oral Maxillofac Surg 2004;33(3):253-57.
3. McNeely ML, Parliament MB, Seikaly H, et al. Effect of exercise on upper extremity pain and dysfunction in head and neck cancer survivors: A randomized controlled trial. Cancer 2008;113(1):214-22.

\section{Chyle Leak:}

1. Nussenbaum B, Liu JH, Sinard RJ. Systematic management of chyle fistula: The South Western experience and review of the literature. Otolaryngol Head Neck Surg 2000;122(1):31-38.

2. Crumley RL, Smith JD. Postoperative chylous fistula prevention and management. Laryngoscope 1976;86(6):804-13.

\section{Rharyngocutaneous Fistula:}

1. De Zinis L, Ferrari L, Tomenzoli D, et al . Postlaryngectomy pharyngocutaneous fistula: Incidence, predisposing factors and therapy. Head Neck 1999:21:131-38.

2. Friedman M, Venkatesan TK, Yakovlev A, et al. Early detection and treatment of postoperative pharyngocutaneous fistula. Otolaryngol Head Neck Surg 1999;121:378-80.

3. Qureshi SS, Chaturvedi P, Pai PS, et al. Prospective study of pharyngocutaneous fistulas following total laryngectomy. J Cancer Res Ther 2005;1(1):51-56.

\section{Flap Complications:}

1. Macnamara M, Pope S, Sadler A, et al. Microvascular free flaps in head and neck surgery. The Journal of Laryngology and Otology 1994:108;962-68.

2. Bui DT, Cordeiro PG, Hu Q, et al. Free Flap Re exploration: Indications, Treatment, and outcomes in 1193 free flaps. Plastic and reconstructive surgery 119;2092-99.

3. Genden EM, Rinaldo A, Suarez C, et al. Complications of free flap transfers for head and neck reconstruction following cancer resection. Oral oncology 2004:40;979-84. 